\title{
Liderazgo transformacional y gestión docente en el marco del estándar tres del modelo del SINEACE en educadores de una IE de Lima
}

\author{
Elizabeth del Carmen Velásquez Fuentes \\ elizabethdelcarmenvelasquezfue@gmail.com
}

ORCID: 0000-0003-2235-1904

Universidad Cesar Vallejo - Perú

\section{RESUMEN}

El presente estudio tuvo como objetivo determinar la incidencia del Liderazgo transformacional del director en la gestión docente en educadores de una IE de Lima. El nivel del estudio es explicativo y de enfoque cualitativo, también se ha seguido un diseño no experimental - transversal. La población objetivo corresponde a los docentes de la institución educativa en estudio, sobre la que se pretende generalizar las características del liderazgo transformacional. Los resultados demostraron que el estudio sirve como base para determinar la importancia del rol del docente líder en el logro de los objetivos de enseñanza - aprendizaje, paralelamente en contribución con el director de la IE. En la matriz para la acreditación, en el estándar tres se desarrolló el estilo de liderazgo colaborativo que asegure el establecimiento y mantenimiento de una visión institucional común, y que promueva un clima favorable para la adecuada organización y articulación de nuestras las funciones para dar soporte a la mejora del proceso de enseñanza aprendizaje, asegurando que los miembros de la comunidad educativa conozcan sus roles y funciones dentro de la organización y su contribución a la mejora del proceso enseñanza-aprendizaje. Se concluyó con la identificación de los roles de liderazgo de los docentes para que tengan una actitud de apertura hacia la introducción de posibles cambios e innovaciones para corregir y mejorar la enseñanza de los alumnos, que trabajen en equipo y sean capaces de tomar decisiones que se direcciones a la calidad en los procesos de aprendizaje.

Palabras clave: liderazgo; transformacional; gestión; docente; estándar; modelo; SINEACE. 


\title{
Transformational leadership and teaching management within the framework of standard three of the SINEACE model in educators of an IE in Lima
}

\begin{abstract}
The objective of this study was to determine the incidence of the director's transformational leadership in teacher management in educators of an EI in Lima. The level of the study is explanatory and with a qualitative approach, a non-experimental cross-sectional design has also been followed. The target population corresponds to the teachers of the educational institution under study, on which it is intended to generalize the characteristics of transformational leadership. The results showed that the study serves as a basis to determine the importance of the role of the teacher leader in achieving the teaching-learning objectives, in parallel with the contribution of the director of EI. In the matrix for accreditation, in standard three the collaborative leadership style was developed that ensures the establishment and maintenance of a common institutional vision, and that promotes a favorable climate for the adequate organization and articulation of our functions to support the improvement of the teaching-learning process, ensuring that the members of the educational community know their roles and functions within the organization and their contribution to the improvement of the teaching-learning process. It was concluded with the identification of the leadership roles of teachers so that they have an open attitude towards the introduction of possible changes and innovations to correct and improve the teaching of students, that they work as a team and are capable of making decisions that are directions to quality in learning processes.
\end{abstract}

Keywords: leadership; transformational; management; teacher; standard; model; SINEACE.

Artículo recibido: 30 noviembre. 2021

Aceptado para publicación: 29 diciembre 2021 Correspondencia: elizabethdelcarmenvelasquezfue@gmail.com

Conflictos de Interés: Ninguna que declarar 


\section{INTRODUCCIÓN}

En el ámbito internacional, nuestro tiempo vive contradicciones fundamentales. Mientras hay difíciles avances en el camino hacia la mayor participación y la expansión democrática, también se encuentran produciendo repercusiones hacia problemas que se creían superados como es el caso que en algunas instituciones se continúe adoptando estilos de liderazgo que no se adecuan a la nueva forma de gestionar. Este es el caso que aborda el siguiente plan, de comprobar cómo las diversas dimensiones del Liderazgo Transformacional se relacionan e inciden en la gestión docente de una institución educativa de Lima.

Por lo tanto, se debe tomar en cuenta como antecedente, que, en el campo de la organización, actualmente se vienen planteando nuevas formas de trabajo que producen cambios en el tipo y forma de liderazgo los cuales priorizan el trabajo en equipo y una gestión flexible que promueva iniciativas y que desarrolle la autocrítica.

Tratar el tema de la gestión educativa es diverso y complejo. Diverso, porque hay diferentes enfoques que nos permiten desarrollar este tema, por la diversidad de actores que se involucran, los que al interrelacionarse determinarán la calidad del producto y del servicio que brindan. El Ministerio de Educación define al PDI, ahora PEI, como "parte de un propósito colectivo donde el director, los profesores y los demás miembros de la comunidad educativa proponen cambios progresivos, importantes y necesarios en el centro educativo y se comprometen a realizarlos" (Ministerio de Educación1997, p. 15). Como vemos, ya desde hace más de 15 años se vienen adoptando nuevas formas de gestionar y para ello es necesario que los directores y docentes adopten y apliquen herramientas modernas de gestión.

En el caso del Liderazgo Transformacional, el propósito de la investigación es medir como esta variable se relaciona con las demás dimensiones de la gestión docente; mejorar la equidad y proveer una educación sensible a las diferencias que discrimine en favor de los más pobres y vulnerables; mejorar la calidad de la enseñanza, aumentar las exigencias y focalizar la atención en los resultados del aprendizaje; profesionalizar el trabajo docente; descentralizar y reorganizar la gestión educativa y ofrecer más autonomía a las escuelas; fortalecer la institución escolar para ofrecer mejor capacidad de operación y mayor responsabilidad por sus resultados, fueron las líneas fundamentales que con distinto énfasis pusieron en marcha los gobiernos de la región. 
Desde la década de 1970, la fisonomía de la educación alrededor del mundo ha enfrentado un conjunto transformaciones (Zabalza, 2015). Una transformación destacable en el ámbito educativo responde a dos procesos paralelos: la masificación de las instituciones educativas y la progresiva pluralidad de los alumnos. Ambos fenómenos son el resultado del aumento del número de las instituciones educativas y de la expansión en el acceso a la educación terciaria, en especial en los países desarrollados (Altbach \& Davis, 2012; Zabalza, 2015; López F., 2012; Rama, 2013). Estos dos procesos han quebrado el elitismo que, desde su origen, caracterizó a las instituciones educativas y representarían la transformación mundial más importante de la enseñanza y su gestión (Thorne, 2012). En respuesta a ello, surgieron los sistemas destinados a la evaluación y al aseguramiento de la calidad, como los rankings y la acreditación de las instituciones educativa, los cuales representan el segundo cambio en la fisonomía de la educación (Peace, 2013 y González \& Ayarza, 2013).

Otro elemento de transformación está constituida por la globalización y la internacionalización de la educación, fenómenos que se han plasmado en la transnacionalización de las instituciones educativas, el incremento de profesores que trabajan internacionalmente y el aumento de alumnos que estudian en el extranjero, así como en la homologación de títulos y la estandarización de programas entre diferentes países (Zabalza, 2015, p. 12).

En asociación a la globalización aparece la privatización de la enseñanza, la cual ha sido acompañada por la consolidación de una visión que considera a la educación como una actividad empresarial. En términos más puntuales, algunos autores aluden al afianzamiento del modelo corporativo de gestión educativa; mientras que otros destacan/cuestionan el crecimiento de la actividad comercial en el campus (Zabalza, 2015, p. 11). Ello preocupa por su amenaza a los estándares académicos, la potencial pérdida de la reputación de las instituciones educativas, y el riesgo de que se debiliten la cooperación y la confianza dentro las comunidades académicas.

Como resultado de fenómenos transformacionales dados en el mundo, como es el caso de la globalización, privatización y la corporativización; la competencia entre las instituciones educativas se ha incrementado, evolución que son elementales para afianzar el cambio en las instituciones y poder disputarse estudiantes y sistemas de investigación. Este contexto también genera las condiciones para que, entre las instituciones dedicadas 
a la enseñanza de calidad, sean cada vez más comunes no solo las alianzas estratégicas sino también las fusiones y adquisiciones. La privatización de la enseñanza también ayudaría a explicar el ataque que sufren los regímenes de permanencia laboral de los docentes y el rápido incremento del docente a tiempo parcial.

Otro cambio de la educación es el aumento del uso de las tecnologías de información y comunicación (TICs), las cuales han facilitado el aprendizaje, han comenzado a desplazar a la biblioteca tradicional y han permitido la expansión de las denominadas "instituciones abiertas" (Zabalza, 2015). De modo paralelo, aparecen nuevos tipos de organizaciones académicas y las existentes atienden a grupos más amplios y diversos. Si a ello se suman la progresiva diferenciación y fragmentación disciplinar y la aparición de los campos de estudio interdisciplinar y multidisciplinar, se evidencia el aumento de la complejidad y la heterogeneidad de las instituciones que ofrecen enseñanza de nivel terciario. Así, la octava transformación de la educación se traduce en una mayor diversidad de la oferta académica (Luengo, 2013; Wooldridge, 2012, y López, 2012).

Frente a los cambios que se han mencionado, se hace necesario analizar la condición de liderazgo, la cual se encuentra muy relacionada con el ejercicio de casi todas las profesiones y actividades de la vida diaria al extremo de considerarlo condición esencial en el éxito profesional y político. Él ejercicio profesional de la pedagogía no se encuentra exenta a estos planteamientos, empero en la mayoría de casos, en el campo pedagógico, se lo asocia a la conducción y la dirección, concretamente: a la figura del Director quien es el encargado de dirigir, impulsar y motivar a la comunidad educativa por tanto se considera que debe ser él quien ejerza un liderazgo eficiente y eficaz.

En esta investigación queremos analizar el liderazgo transformacional del director y su relación con la gestión del docente del aula como elemento transformado y determinar cual contribuye de mejor manera a la gestión del docente de aula, esto implica que el docente como tal, debe conocer y ejercer su rol y función de líder en el aula: este rol llevado a cabo eficientemente, influye creando un clima de confianza, de respeto en el aula que permitan bases para el logro de los objetivos del proceso enseñanza-aprendizaje. Los gobiernos, las autoridades educativas y la sociedad exigen líderes y personas integrales, pero eso no se logra sin tener en las aulas con líderes; las corrientes educativas que actualmente están adoptando los sectores educativos, están encaminadas a que el papel del docente sea más activo y transformador dentro del aula. 
Este estudio tiene por finalidad servir de base a futuras investigaciones para desarrollar trabajos aún más específicos sobre el rol y función del docente líder en el aula.

El problema que da origen a esta investigación surge luego de analizar los estándares de la matriz de IPEBA y priorizar los referidos a la acción docente y su gestión como líder en el aula (SINEACE - IPEBA, 2015) orientados al mejoramiento de la calidad de la educación. El estándar tres del modelo de SINEACES se refiere a la ejecución de un estilo de liderazgo que de forma colaborativa asegure un clima favorable. Este estándar se refiere a toda la comunidad educativa en esta investigación se orienta, hacia el actuar del docente en el aula, es decir, la aplicación del estándar y el indicador tres puntos dos en lo referente al rol y función del docente como gestor del liderazgo que contribuya a lograr la mejora en el proceso de enseñanza aprendizaje.

Sin embargo, también consideramos que en la realidad el docente desconoce este rol y su actuación en el aula se convierte en una isla desconectada de los otros actores del proceso educativo: directivos, docentes, estudiantes, padres de familia y personal administrativo. Este actuar dificulta el proceso de cambio y transformación e incrementa el esfuerzo del propio docente, no permite asegurar el establecimiento y mantenimiento de una visión institucional común. Para tener un enfoque preciso del problema se formuló la siguiente interrogante ¿Cómo incide el Liderazgo transformacional del director en la gestión docente en educadores de una IE de Lima?.

\section{ESTRATEGIAS METODOLÓGICAS O MATERIALES Y MÉTODOS}

La investigación es de tipo descriptiva, ex post facto, básica aplicativa. Descriptiva, porque "busca especificar las propiedades, las características y los perfiles importantes de personas, grupos, comunidades o cualquier otro fenómeno que se someta a un análisis" (Sánchez y Reyes, 1992, 32).

También es nivel descriptivo porque reconocemos ciertas características del liderazgo transformacional en una institución educativa de Lima, sobre la base de identificar las relaciones de liderazgo entre el director y los educadores de dicha institución. El nivel descriptivo de investigación implica más que la recolección y tabulación de datos. Significa un elemento interpretativo, significativo y de importancia de aquello descrito (Fernández 1986: 413). Por tanto, buscamos identificar las características del liderazgo en un director y recoger las opiniones de los docentes acerca de las características del liderazgo que posee dicho director de la Institución Educativa. 
En la investigación se sigue el diseño no experimental - transversal. No experimental, porque "no se realizará una manipulación de las variables"; transversal, porque "recopilaran datos en un solo momento dado", y de esta manera identificar los tipos de liderazgo.

La investigación no experimental, "es la búsqueda empírica y sistemática en la que el científico no posee control directo de las variables independientes" (Keringer 2002), no se construye ninguna situación, es más natural y cercana a la realidad cotidiana (Mertens, 2005), que señala que la investigación no experimental es apropiada para variables que no pueden o deben ser manipuladas o resulta complicado hacerlo. Se realizan cuando el investigador toma una muestra de sujetos para ser evaluar en distintos tiempos y por un periodo bastante largo.

\section{RESULTADOS Y DISCUSIÓN}

Existen investigaciones que toman aisladamente las variables objeto de estudio, orientados a la acción de liderazgo del director, o que son aplicadas en niveles distintos al nivel secundario, e incluso investigaciones realizadas más desde el punto de vista de la psicología. Por ello se pretende hacer una investigación que integre ambas variables y en el nivel secundario.

Se estima que el problema de investigación tiene relevancia científica y social porque afecta no sólo a los estudiantes y a los docentes sino a toda la comunidad educativa. Sus resultados son importantes para la realización de nuevas investigaciones en la temática, aportando sugerencias e interrogantes, y abriendo la discusión sobre el significado de ser docente, su rol de líder en el aula y trabajar como tal.

El resultado de estudio ha permitido tomar medidas correctivas para mejorar los niveles de eficiencia del trabajo docente, partiendo de la voluntad de los docentes para mejorar, buscando asesoramiento, enseñanzas, experiencias, consejos y apoyo moral de sus colegas, lo cual implicará un esfuerzo organizado para seleccionar las mejores alternativas disponibles con el fin de conseguir el cumplimiento de metas específicas, lo cual repercutirá en la satisfacción propia del docente. Lo mencionado será de valor extraordinario para los docentes ya que puede transformar una enseñanza difícil en una tarea más llevadera y productiva.

La investigación contribuye culturalmente porque es un enfoque original, porque el liderazgo está direccionado hacia el docente del aula en particular y si bien es cierto que 
es en el aula en donde se ejerce lo sustantivo de la acción educativa, también es cierto que pocas investigaciones tratan el tema por tal motivo de esta investigación se benefician tanto las Instituciones Educativas que deseen iniciar su proceso de autoevaluación como la Institución Educativa de Lima en particular sus Directivos, docentes, alumnos, padres de familia y la comunidad en general. De igual manera el presente estudio podrá servir de base para ampliar y/o profundizar el tema en estudios posteriores.

\section{DISCUSIÓN}

Vásquez (2012) señaló en esta investigación de tipo descriptivo simple, los estilos y dimensiones del liderazgo docente, analizados en una escuela pública de La Perla, Callao; hallando sus niveles de dominio y que han sido establecidos por género y perfil de liderazgo prevalente. El autor expresó lo siguiente: “el cuestionario para identificar estilos de liderazgo (CIELID), validado por jueces; es una adaptación del MLQ 5X de Vega y Zavala (2017), y del CELID de Castro et al. (2007); para las dimensiones que se catalogaron como "Seguidor" su validación fue 0,93 ; y la fiabilidad 0,85; para la forma Líder, la fiabilidad fue 0,83 . La muestra de 18 profesores respondieron a la forma Líder autoevaluativa; y los 90 estudiantes de secundaria del cuarto y quinto grado, a la forma Seguidor, evaluando a dichos profesores. También Vásquez encontró "un dominio medio de los estilos y dimensiones de liderazgo; predominó el estilo transaccional; recompensa contingente e inspiración fueron las dimensiones prevalentes; las docentes obtuvieron mayores puntuaciones en el estilo transformacional, en las dimensiones transformacionales y en la recompensa contingente; los varones, en los estilos transaccional y laissez faire, y en la dirección por excepción; el perfil de liderazgo fue transaccional, no óptimo" (p. 12).

Fernández (2018) realizó la investigación titulada "Desempeño docente y su relación a la meta, estrategias de aprendizaje y auto eficiencia: un estudio con maestros de primaria de Lima, Perú", el objetivo fue "estudiar la relación existente entre la orientación a la meta, estrategias de aprendizaje, autoeficacia y la percepción del desempeño docente en profesores de Educación Primaria de Lima Metropolitana. La muestra estuvo conformada por 313 maestros de las diferentes UGEL (es) de Lima Metropolitana, de los cuales 224 son del sexo femenino y 89 del sexo masculino". Los instrumentos utilizados en el estudio fueron: el cuestionario de Orientación a la Meta del Profesor (PALS, 1997, 2000), el Cuestionario Estrategias de Aprendizaje y Metacognitiva (MSQL, 1991), la Escala de 
Eficacia Percibida de los maestros de Tschannen-Moran y Woolfok (2014) y el Cuestionario de Autorreporte del Desempeño Docente (Fernández, 2002). Los resultados indicaron la presencia de una relación efectiva entre las variables analizadas. Asimismo, se identificó la influencia de la variable prácticas de aprendizaje del maestro, sobre el desempeño docente según los niveles de autoeficacia y las estrategias de aprendizaje. Se discuten los resultados en función de los objetivos del estudio.

Por su parte Flores (2003) realizó un referido a los estilos de liderazgo y su relación con el desempeño docente en el aula, según el conocimiento de los alumnos del quinto grado de secundaria, en los colegios estatales de áreas técnicas de la USE Nº 06 de Ate-Vitarte. De una población de diez colegios, se seleccionó una muestra de cinco al azar. Se utilizó el cuestionario de descripción del comportamiento del líder, propuesto por House y Desler (1974). En dicho estudio, se logró determinar los estilos de liderazgo predominantes: Tolerancia a la libertad, consideración, énfasis en la producción e iniciación de estructura; así como los niveles y áreas de desempeño docente en el aula: didáctica, personalidad, motivación, orientación y habilidades para la enseñanza. Las conclusiones indicaron que solo existe relación entre los estilos de liderazgo tolerancia y consideración cada uno independiente del desempeño del docente en el aula.

Cano, Hernández y Aguilar (2013) señalaron en su investigación que el liderazgo que asumen las personas que están frente a un determinado grupo de personas puede determinar en gran medida el accionar de ese mismo grupo; en el caso de los docentes les ha tocado desempeñar un papel de trascendencia en el aula, el liderazgo que ejercen de forma natural puede conducir a resultados importantes en el desempeño escolar de los estudiantes. Las instituciones escolares determinan su política interna, y dentro de esta se puede encontrar que el liderazgo del docente se convierte en una estrategia que puede ayudar al logro de las metas planteadas por cada organización escolar; sin embargo, dentro de la política interna de cada institución educativa no es común apreciar libertad plena a cada docente para que este sea líder y un gran influyente en la conducta de los estudiantes, por el contrario el accionar de los maestros está regulado y vigilado por el director de la institución educativa. Por lo tanto, son los alumnos los que deben opinar sobre el interés que tiene el liderazgo de los docentes.

También se encuentra la investigación de Maureira y Rojas (2013) sostuvieron que el liderazgo colectivo, distribuido o compartido, emerge como propuesta para enriquecer, 
desde fuentes no tradicionales de influencias, el conocimiento, la formación y la mejora de la calidad de la intervención en los centros escolares. El presente estudio describe el liderazgo transformacional de los docentes de lenguaje y comunicación de 7 básico a 4 año de medio en cinco establecimientos escolares que atienden a niños y jóvenes de escasos recursos. La descripción se basa en los resultados de la aplicación del cuestionario multifactorial de liderazgo a una muestra de 505 estudiantes. A través de parámetros de estadística descriptiva y el test de la prueba de t-student, se evidencia la inexistencia de diferencias estadísticamente significativas en la percepción del liderazgo del docente de lenguaje, según ciclos de enseñanza, como asimismo que los docentes de lenguaje son percibidos con rasgos transformacionales.

Horn y Marfán (2015) desarrollaron la investigación titulada "Relación entre el liderazgo educativo y desempeño escolar: revisión de la investigación en Chile", los investigadores en su resumen señalan dar cuenta de 14 estudios sustentados en el país de Chile, el sustento ha sido empírico ya que rescatan la importancia del liderazgo educativo en el logro de buenos resultados escolares. Los estudios analizados han sido producto de una revisión bibliográfica de publicaciones del año 2000 en adelante. El objetivo de la investigación fue presentar los alcances de la investigación empírica que se ha desarrollado, sobre la relación entre liderazgo escolar y resultados de aprendizaje en el sistema escolar chileno. "De manera resumida se muestra la evidencia que se ha ido desplegando durante los últimos 10 años de exploración, de manera paralela, se desarrollaron metodologías para poder plantear sus resultados y conclusiones". Finalmente, se ubican en diálogo los avances y falencias que muestra la investigación avanzada hasta la fecha en el tema, así como su sintonía con los avances de la investigación a nivel mundial.

Por su parte Bolívar (2015) señala en su estudio lo siguiente "la literatura sobre eficacia y mejora de la escuela ha destacado el papel crítico que ejerce la dirección en organizar buenas prácticas pedagógicas en los centros educacionales e incrementar los resultados del aprendizaje. Se hace una revisión actual de las investigaciones sobre cómo el liderazgo educativo es un factor de primer orden en la mejora de resultados". "Hasta ahora, tanto en España como en Chile, la dirección ha tenido escasa capacidad para incidir en la mejora de las prácticas docentes; sin embargo, las nuevas orientaciones de la investigación y de las políticas educativas potencian el liderazgo pedagógico. El perfil 
directivo de gestión se está, pues, ampliando a un liderazgo pedagógico. Un liderazgo para el aprendizaje, más allá de la dirección, se tiene que ampliar, de modo distribuido, a través del liderazgo del profesorado y de las comunidades profesionales de aprendizaje. Se discute igualmente las graves limitaciones que la dirección actual tiene para diseñar entornos de mejora del aprendizaje de todos los alumnos".

Pedraja (2009) señala que, en el desarrollo de esta investigación, se estudió la existencia o no de una relación estadísticamente significativa entre los estilos de liderazgo y los resultados que los estudiantes obtienen en la prueba nacional del sistema de medición de la calidad de la educación. Para este efecto se trabajó con una muestra de 21 escuelas de enseñanza básica de la ciudad de Arica, Chile. Las Conclusiones abordan en sus resultados que el estilo de liderazgo transformacional influye positivamente sobre la calidad de los colegios, el estilo transaccional no tiene efecto estadísticamente significativo, en tanto que el estilo "laissez faire" afecta negativamente los resultados en el sistema de medición de la calidad de la educación.

Por otro lado, Cuadra (2007) señala que el liderazgo es uno de los temas que más interés suscita en el ámbito de la psicología social de las organizaciones, generando un cúmulo importante de investigación. Dentro de los hallazgos relevantes que se encuentran en la literatura científica especializada, se tiene el impacto significativo que el liderazgo ejerce sobre variables asociadas a resultados organizacionales, como satisfacción y clima laboral. Considerando lo anterior, el presente estudio tiene como propósito confirmar, en una muestra local (Arica), las relaciones de liderazgo transformacional, transaccional, consideración e iniciación de estructura con satisfacción y clima organizacional. Los resultados dan cuenta de las relaciones entre liderazgo y las variables dependientes. Las conclusiones indican que el objetivo inicial de este trabajo era comprobar las relaciones existentes entre variables asociadas al liderazgo (transformacional, transaccional, consideración e iniciación de estructura) e indicadores subjetivos de satisfacción y clima. Cada una de las relaciones hipotetizadas se confirmaron mediante los análisis estadísticos correspondientes. Por tanto, se puede establecer que, liderazgo ejerce una influencia positiva y significativa en variables como satisfacción y clima laboral, encontrándose que el liderazgo explica el $49 \%$ de la varianza del clima general y un 31\% de la varianza de la satisfacción laboral, lo cual no constituye un hecho menor, siendo, una de las variables que más peso tiene en la organización. 
Salazar (2019) indica que la educación constituye un proceso simultáneo, tanto de construcción de nuevos saberes y desarrollo personal, lo que exige en el mundo contemporáneo una práctica de la gestión educativa interactiva y participativa, en que el directivo, como impulsador de la innovación y del cambio permanente, debe intervenir como sujeto activo que aproveche el recurso humano de la institución, para que sea partícipe en los diferentes roles a los cuales debe atender para lograr los objetivos propuestos sin mayores dificultades, buscando adaptarse a situaciones cambiantes.

En base a estos señalamientos, es importante destacar que las instituciones educativas, deben ser gerenciadas por individuos cuyas cualidades y habilidades profesionales, estén vinculadas a la comunicación, motivación y establecimiento de las relaciones interpersonales dentro de una gestión educativa, en la cual tiene un rol protagónico las relaciones humanas.

De lo expuesto anteriormente, se enfatiza la necesidad de que el gerente educativo de las instituciones, cumpla a cabalidad sus funciones destacando entre ellas el liderazgo transformacional como medio para desarrollar una gestión educativa con una misión y visión organizacional compartida, que le ayuden a planificar su trabajo y a la vez le sirva para controlar, orientar, dirigir y evaluar a los docentes y demás miembros que participan en el proceso educativo como partes implicadas que apoyan a las instituciones escolares hacia una transformación social y educativa, tomando en cuenta que el éxito de una organización depende del crecimiento y desarrollo de quienes la integran. Las conclusiones, señalan que la gerencia actual que se realiza en las instituciones educativas son objeto de estudios, pues la misma según los hallazgos producto de la investigación realizada está inmersa en modelos gerenciales tradicionales, lo cual implica que no se ofrecen las soluciones a los problemas o situaciones que se suponen están presentes en las organizaciones y de no darse un cambio en las mismas, la situación crítica gerencial seguirá imperando en ellas, limitándose así la posibilidad de conocer y asimilar de las transformaciones y evoluciones de los nuevos tiempos. En consecuencia, un gerente educativo debe estar consciente de las necesidades y motivaciones de su personal en función de las características comunes del grupo organizacional, para dirigir eficientemente la institución tomando en cuenta que la toma de decisiones y la delegación de autoridad es fundamental dentro de una organización. 
El personal directivo debe establecer y poner en práctica la combinación de los diferentes sistemas de comunicación con todo su personal, se logre la integración, a fin de asegurar un trabajo coherente encaminado hacia objetivos comunes que despierten la iniciativa e innovación en el trabajo docente. Se debe respaldar y motivar al personal por medio de los diferentes tipos de comunicación, crear un clima propicio de confianza y cooperación que elimina el aislamiento y en consecuencia desaparecer la angustia y la confusión entre los integrantes de la organización, permitiendo mayor fluidez en la comunicación. Partiendo de lo expuesto, no debe negarse el inmenso privilegio otorgado a la comunicación como función permanente, esencial es inherente a la naturaleza humana y es un factor esencial de convivencia y un elemento determinante de las formas que asumen la socialización del individuo.

Por otro lado, en la matriz para la acreditación, en el estándar tres se indica lo siguiente: "Desarrollemos un estilo de liderazgo colaborativo que asegure el establecimiento y mantenimiento de una visión institucional común, y que promueva un clima favorable para la adecuada organización y articulación de nuestras las funciones para dar soporte a la mejora del proceso de enseñanza - aprendizaje” (SINEACE - IPEBA, 2015)

Razón por la cual se señalan cuatro indicadores resultantes de este estándar. El indicador 3.1 señala: “Asegurarnos que los miembros de la comunidad educativa conozcan sus roles y funciones dentro de la organización y su contribución a la mejora del proceso enseñanza-aprendizaje" (IPEBA, 2014)

En este indicador habla del conocimiento que debe tener el docente de su rol y función de líder en el aula. Por lo tanto la Matriz de IPEBA establece la importancia del rol y función del docente como líder en el aula. Este rol tiene como objetivo el logro del proceso enseñanza - aprendizaje, entre otros.

El indicador 3.2 señala "Implementamos mecanismos para articular los roles, funciones y responsabilidades de todos los miembros de la comunidad educativa para dar soporte a la mejora del proceso enseñanza aprendizaje” (IPEBA, 2014)

En este indicador se enfatiza que todos los miembros de la comunidad educativa se deben encontrar comprometidos en el cumplimiento de sus roles y funciones que no tienen otra finalidad que coadyuvar al logro de los objetivos del proceso enseñanza aprendizaje, en este caso los directivos, docentes, personal administrativo y de servicio así como los padres de familia, deben asumir el compromiso de apoyar el liderazgo del docente de 
aula; la investigación trata de auscultar en qué medida el docente es capaz de gestionar, y articula a tos los actores de la Institución educativa a fin de lograr los objetivos El indicador 3.3 señala: "Implementamos estrategias para desarrollar un clima institucional de confianza y respeto que promueva el bienestar y buen desempeño de todos los miembros de nuestra comunidad educativa”. (IPEBA, 2014).

En este indicador se incide en el rol y función del docente como propiciador y gestor de un clima de aula basado en la confianza y respeto mutuo, entre otros, esto justifica una vez más la importancia del liderazgo docente para lograr el compromiso para que todos los estamentos apoyen al docente, sobre la base de que el clima de bienestar propicia el buen desempeño del alumno en el aula.

El liderazgo transformacional es un tipo de liderazgo incorporado recientemente en las organizaciones educativas debido a la promoción de relaciones interpersonales entre los directores y los docentes. Algunos autores como James Burns (1978) y Bernard Bass (1985) presentan estilos muy marcados enfatizando perspectivas diferenciadas, ambos consideraron, en sentido amplio, que el liderazgo transformacional se complementaba con el liderazgo transaccional en sus interrelaciones entre líderes y seguidores.

Según Yukl describió el liderazgo sobre como: “.... a stream of evolving interrelationships in which leaders are continuously evoking motivational responses from followers and modifying their behavior as they meet responsiveness or resistance, in a ceaseless process of flow and counterflow" (Yukl 1989a: 210).

Percibimos el liderazgo transformacional desde una doble dinámica entre los niveles micro y macro. En el nivel micro influyen los procesos individuales mientras en el nivel macro influyen en la movilización del poder en función del cambio del sistema social y la reforma de la institución. Retomaremos las palabras de James MacGregor Burns citado por Yukl, quien describió el liderazgo transformacional como un proceso en el cual: leaders and followers raise one another to higher levels of morality and motivation" (Yukl 1989a: 210).

La idea de Burns consideró tanto a los líderes transformacionales como a sus seguidores sujetos que buscaban desarrollar su conciencia, lo cual conduce a un incremento de la moralidad y la motivación en sus acciones; esto se refuerza haciendo atractivos los ideales de libertad y justicia para una mejor motivación laboral, pero jamás las emociones del temor o los celos para conseguir motivarlos en sus obligaciones. Así, la moralidad es 
importante en el proceso de mutuo desarrollo de la conciencia con fines de lograr una relación vinculante entre el líder y sus seguidores.

Burns, citado por Gary Yukl (1989b: 260), consideró que el liderazgo transformacional se vincula con los valores morales en forma positiva y con las necesidades de los seguidores. Para Bass, citado por Yukl (1989b: 260), el líder motiva al seguidor e incrementa su sentido de obligación, pero no se preocupa por los beneficios a favor de los seguidores.

Además, existen otras concepciones en las características que presenta el liderazgo transformacional. Nos apoyaremos en la tesis de Carolina Vega Villa y Gloria Zavala Villalón para optar el título de psicóloga titulada “Adaptación del Cuestionario Multifactorial de Liderazgo MLQ (versión 5 X Corta) de Bernard.

Por nuestra parte, consideramos como características en la práctica del liderazgo transformacional: la capacidad de negociar, el control de los resultados, la capacidad de construir una visión atrayente al colaborador y comunicarla apropiadamente para convencer a dicho colaborador. El líder transformacional es al mismo tiempo carismático y visionario porque es capaz de convencer a sus seguidores en necesidades de reconocimiento planteadas por él. Por tanto, los líderes (transformacionales) utilizan al máximo sus fortalezas para minimizar sus puntos débiles y desarrollan comunicación eficaz obteniendo apoyo y cooperación de sus seguidores (Cardona 2000: 8).

\section{CONCLUSIÓN O CONSIDERACIONES FINALES}

- La investigación es de gran motivación para que el docente, se desempeñe con éxito en cualquier escenario de la vida, para lo cual se hace necesario contar con un perfil docente centrado asumiendo la docencia como una profesión que implica fundamentarse en lo más valioso del patrimonio cultural de la humanidad y ejercer la actividad docente con eficiencia, espíritu de transformación y cambio.

- En nuestro contexto el liderazgo ejercido por el director de la I.E. Pública tiene una gran relevancia por ser quien dirige los procesos de participación en el interior de su institución educativa. Es necesario enfatizar a la persona del director-líder quien vincula a la comunidad educativa en un proyecto de mejora desde la perspectiva del cambio (Murillo: 2003: 18) mediante su apertura a las intervenciones positivas de sus docentes. El director de la I.E. Pública debe proponer las estrategias necesarias promotora de participación en sus docentes. Para ello determina el esfuerzo docente 
a realizar, motiva la percepción de justicia en las tareas asignadas y establece criterios de desempeño tanto individual como colectivo (Pedraja; Rodríguez y Rodríguez 2006: 578) a fin de establecer una cultura organizacional sana.

- Las instituciones educativas y empresas en general de hoy en día están centrando su atención en el liderazgo como una ventaja competitiva, donde se establece que el éxito o fracaso de cualquier organización dependerá de la calidad de sus líderes. Uno de los activos más importantes de las instituciones ya sean públicas o privadas son las personas, por lo que el líder debe saber conducir, guiar, motivar e integrar al personal dentro de un ambiente de comunicación interpersonal, generando así un clima laboral favorable en la organización, que permita que sus trabajadores entre sí puedan llegar a un punto de vista común donde puedan comprender y cooperar para lograr los objetivos y fines institucionales.

- Esta interpretación de la teoría nos lleva a la justificación del presente trabajo en el campo práctico porque al llevar a cabo el proceso de autoevaluación en lo referente a este estándar se diagnosticará el liderazgo efectuado en la Institución Educativa de Lima, se obtendrán resultados que permitan analizar situaciones y aplicar los planes de mejora. Así mismo, la Institución Educativa que nos facilita la ejecución de esta investigación contará con los resultados obtenidos, contribuyendo a la ejecución de su autoevaluación en lo referido al estándar número tres.

- El problema en cuestión, ha sido materia de preocupación en todos los niveles del sistema educativo, especialmente, en el nivel secundario, donde existe una variedad de factores que aún no han sido tratados con el grado de profundidad y amplitud que requieren, e incluso en algunos casos no se ha estudiado su interrelación, ni se los ha identificado.

- El interés por el tema investigado es identificar, a partir de los propios docentes, que tipo de liderazgo ejercen y como a través de él participan en la transformación y mejoramiento de la calidad educativa. Así mismo centrar la atención de las opiniones de los docentes con el objeto de determinar si sienten o no satisfacción en su trabajo y ver en qué forma se podrían visualizar aquellos factores que están influyendo en dicha satisfacción, así como establecer la interrelación con el desempeño docente y aportar a la creación de los estándares de desempeño docente. 


\section{LISTA DE REFERENCIAS}

UCCOR. (12 de Agosto de 2015). Factores de insatisfacción laboral en los docentes de $E G B$. From http://www.uccor.edu.ar/paginas/REDUC/grasso.pdf

Zabalza, M. (7 de Diciembre de 2015). Didáctica universitaria. Conferencia pronunciada en la Pontificia Universidad Javeriana de Cali, el 9 de febrero de 2005. From http://portales.puj.edu.co/didactica/Archivos/Didactica/DIDACTICAUNIVERSI TARIA.pdf

Valdes, H. (2009). Manual de buenas prácticas de evaluación del desempeño profesional de los docentes. Lima: Consejo Nacional de Educación. Perú.

Vásquez, D. (2012). Estilos de liderazgo de los docentes del nivel secundaria de una Institución Educativa del distrito de La Perla Callao. Callao: Tesis USIL.

Altbach, P., \& Davis, T. (2012). Desafío global y respuesta nacional. Notas para un diálogo internacional sobre educación superior. Buenos Aires: Biblos.

Atalaya, M. (2011). Satisfacción Académico-Administrativa en Estudiantes de Postgrado. Lima: UNMSM.

Báez, M., Cazenave, M., \& Lagos, J. (2018). Percepción de la evaluación del desempeño docente: diagnóstico desde la perspectiva del docente. Santiago de Chile: Grupo Interuniversitario de Desarrollo.

Baeza, J., Pérez, M., \& Reyes, L. (2009). Estándares de Desempeño Docente. Sistematización de Contenidos para su operacionalización. Chile: Ediciones Universidad Católica Silva Henríquez.

Balzán, Y. (12 de Diciembre de 2018). Acompañamiento Pedagógico del Supervisor y Desempeño docente en las escuelas de III etapa de básica. From Maracaibo.intercambio pedagógico.mht

Bass \& Avolio. (1993). Liderazgo Transformacional. Lima: Centrum.

Bolivar, A. (2015). El liderazgo educativo y su papel en la mejora: una revisión actual de sus posibilidades y limitaciones. Scielo, 33 .

Cuenca, R., Ames, P., Córdova, S., Guerrero, L., Iguiñiz, M., Muñoz, F., et al. (2012). La educación en los tiempos del APRA: Balance 2006- 2009. Lima: Foro Educativo.

Canal, J. (2013). Ingresos y satisfacción laboral de los trabajadores españoles con título de doctor. Rev.Esp.Investig.Sociol. ISSN-L: 0210-5233. $N^{o}$ 144, Octubre Diciembre 2013, pp. 49-72, 34. 
Cano Flores, M., Hernández Rodríguez, C., \& Aguilar, D. V. (2013). Diagnóstico sobre el liderazgo docente ¿Estrategia de la micropolitica en las organizaciones escolares de educación superior? Mexico: Ciencia Adminsitrativa.

Carbajal, J. (2013). Clima organizacional y satisfacción del docente en la Universidad de Trujillo. Trujillo: UNT.

Chíavenato, I. (2015). Administración de Recursos Humanos. Colombia: Edit. Mc Graw Hill.

Chile, M. d. (2014). Estándares de desempeño. Santiago de Chile: Programa de Fortalecimiento de la Formación Inicial Docente.

Colina, Z. (2018). Modelo para la evaluación del desempeño docente en la función docencia universitaria. Lima.

CONEASES. (20145). CONEASES. Lima.

Cortez, R. (2006). Influencia Del Liderazgo De Los Directivos Y Jerárquicos En La Satisfacción Laboral De Los Docentes Y Administrativos De La Institución Educativa “Manuel A. Odria” Del Distrito De Ciudad Nueva, En El Año 2006. Tacna: Tesis de Licenciado en Educación Técnica.

Evans, J. (1998). Teacher morale,Job satisfaction and motivation. London: Paul Chapman Publishing ltda. .

Evkall, G., \& Ryhammar, L. (2014). Leadership style social climate and organizational outcomes: A study of a Swedish University College. New York: Creativity and Innovation Managemen.

Eduxere. (24 de Octubre de 2014). La Evaluación Docente. From http://eduxere.blogspot.pe/2007/10/niveles-de-desempeo.html

Eisenhardt, K., \& Bourgeois, L. (2012). Politics of strategic decision making in high velocity environments: Toward a midrange theory. New York: Academy of Management Journal,.

Estrada, L. (2015). El desempeño docente. Venezuela: Facultad de Ciencias de la Educación, de la Universidad de Carabobo.

Delano, N. (1991). Satisfacción laboral y estrés en profesores de educación básica general. Tesis para optar grado de licenciado en ciencias de la educación. Santiago de Chile: Tesis para optar grado de licenciado en ciencias de la educación. 
Díaz, H., \& Saavedra, L. (2012). a carrera del maestro en el Perú. Lima: Grade.

Dipp, J., Villanueva, R., \& Salas, M. (19 de Octubre de 2011). Desempeño Docente y Satisfacción Laboral en Educación Superior. From http://www.repositoriodigital.ipn.mx/handle/123456789/2715

Fernandez Arata, J. (2018). Desempeño docente y su relación con orientación a la meta, estrategias de aprendizaje y autoeficacia: un estudio con maestros de primaria de Lima, Perú. Lima: Instituto de Investigación de Psicología, Universidad de San Martín de Porres, Perú.

Ferrer, P. (23 de Enero de 2013). Estándares y competencias. From https://metodoevaluacion.wordpress.com/estan-desem/

Guizar, R. (1998). Desarrollo organizacional. Principios y Aplicaciones. México: Mc Graw Hill.

González, L., \& Ayarza, E. (17 de Noviembre de 2013). Calidad, evaluación institucional y acreditación en la educación superior en la región Latinoamericana y del Caribe. From www.imasde.cl/doc/gestioninstitu/Calidad.pdf

Ivancevich, J., Lorenzi, P., \& Crosby, P. (2012). Gestión Calidad y Competitividad. Lima: McGraw-Hill.

IPEBA. (2014). Matriz de evaluación de la Calidad de la Gestión de Instituciones de Educación Básica Regular. Lima, Perú: SINEACE.

Iturra, G. (1998). Relación de la dependencia administrativa con la satisfacción laboral en profesores básicos de Temuco. Chile: Tesis para obtener grado de magister en educación. Mención evaluación. Universidad la Frontera.

Horn, A., \& Marfán, J. (2015). Relación Entre Liderazgo Educativo y Desempeño Escolar: Revisión de la Investigación en Chile. Scielo , 104.

Ley del SINEACE. (2006). Ley del SINEACE. Articulo 11 (2006). Lima.

Ley General de Educación. (2003). Ley General de Educación. Articulo 13. Lima.

Ley General del SINEACE. (2006). Lima: Sineace.

López, F. (2012). Notas para un estudio comparado de la educación superior a nivel mundial. Buenos Aires: Consejo Latinoamericano de Ciencias Sociales CLACSO. Escenarios mundiales de la educación superior: Análisis global y estudio de casos ( $1^{\mathrm{a}}$ ed.) (pp. 21-105). . 
López, N. (2017). Educación y equidad. Algunos aportes desde la noción de Educabilidad. Buenos Aires: Instituto Internacional de Planeamiento de la Educación.

Lorenzo, M., \& Sáenz, O. (2011). Evaluación de la satisfacción del profesorado universitario. En Actas del I Congreso Internacional sobre calidad de Enseñanza Universitaria. . Granada: ICE, Universidad de Cádiz.

Nolazco, E. (2012). Autopercepción del desempeño docente y satisfacción laboral en una Institución Educativa en el Perú. La Molina: Fcultad de Educación USIL.

Murillo, J., \& Roman, M. (2013). Docentes en educación primaria en América Latina con más de una actividad laboral. Redalyc.org , 58.

Murillo, J., \& Roman, M. (2013). Docentes en educación primaria en América Latina con más de una actividad laboral: situación e implicaciones. Scielo. Universidad Autónoma de Madrid. , 15.

Maureira, O. (2017). Liderazgo y eficacia escolar: Hacia un modelo causa (Vol. 2). Chile: REICE Revista Iberoamericana sobre Calidad, Eficacia y Cambio en Educación.

Maureira, O., \& Rojas, A. (2013). Características del Liderazgo Transformacional en Docentes de Lenguaje y Comunicación. Chile: Universidad Católica Silva Henríquez.

Ministerio de Educación. (2005). Ley General de Educación. Articulo 49. Lima.

Oficina Regional de Educación para América y el Ca. (2012). Evaluación del desempeño y carrera profesional docente. Unesco, 231.

Ortiz, P., \& Cruz, L. (2014). Estudio sobre clima y satisfacción laboral en una empresa comercializadora. Psicologia para América Latina, 32.

Quipe, J. (2013). El Clima organizacional y desempeño de los docente en la institución educativa 31507 Domingo Faustino Sarmiento de la provincia de Huancayo2011. Trujillo: Universidad Antenor Orrego.

Paúcar, E. (2018). Teorías motivacionales de proceso y desempeño docente en el Instituto Superior Pedagógico Público Huaraz. Huaraz: Tesis.

Palma, S. (1999). Elaboración y validación de una escala de satisfacción laboral en trabajadores de Lima Metropolitana. Lima: Revista e investigación en psicologia. 
Peace, M. (12 de Junio de 2013). Strengthening World Bank support for quality assurance and accreditation in higher education in East Asia and the Pacific. From UNESCO: Second Global Forum on International Quality Assurance, Accreditation and the Recognition of Qualifications in Higher Education "Widening Access to Quality Higher Education", Paris, 28 - 29 Junio de 2017.: http://portal.unesco.org/education/en/files/31954/10879919543INF9ENG-

Peiro, J. (2012). Tratado de Psicología del trabajo. Madrid: Vol. I. Actividad laboral en su contexto.

Peña, J., \& Liarzaburu, L. (2011). Desempeño docente en la Universidad Privada "Sergio Bernales”, Cañete (Perú): una aproximación desde la perspectiva del docente. Lima: Magister consultores asociados.

Subaldo, L., Gómez, L., Gonzáles, J., \& Martínez, A. (2012). Las repercusiones del desempeño docente en la satisfacion y el desgaste del profesorado. RODERIC, 241.

Salluca, L. (2015). Relación entre niveles de satisfacción laboral y desempeño docente en Instituciones Educativas del Cercado Callao. Lima: USIL.

SINEACE - IPEBA. (8 de Diciembre de 2015). Matriz de Evaluación de la Calidad de la Gestión de Instituciones de Educación Básica Regular. From http://www.sineace.gob.pe/wpcontent/uploads/2014/08/Matriz_EBR_Actualizada_.pdf

Stoner, J. (2012). Administración. México: DF: Editorial Prentice- Hall Hispanoamericana.

Rama, C. (4 de Noviembre de 2013). La tercera reforma de la educación superior en América Latina. - From http://www.udlap.mx/rsu/pdf/3/ LaTerceraReformadelaEducacionSuperiorenAmericaLatina.pdf

Reyes Ochoa, L. A. (2015). Estándares de Desempeño Docente. Lima: Ediciones Universidad Católica Silva Henríquez.

Reglamento de la Ley del SINEACE. (2007). Reglamento de la Ley del SINEACE. Articulo 12.1. Lima.

Robbins, S. (2014). Comportamiento organizacional. San Diego: Pearson Educación.

Robbins, S., \& Judge, T. (1999). Comportamiento organizacional. México: Pearson Prentice Hall. 
Rojas, J. (1995). Satisfacción laboral en una muestra de profesores de educación diferencial de la comuna de Nuñoa. Tesis para optar al grado de magister en educación especial. Santiago de Chile: PUCCH.

Thorne, C. (22 de Enero de 2012). Indicadores de calidad de la universidad a nivel internacional y el caso peruano. . From http://sicevaes.csuca.org/drupal/ $? \mathrm{q}=$ filemanager/active $\&$ fid $=234$ 